\title{
One-step fabrication of microfluidic chips with in-plane, adhesive-free interconnections
}

Sabourin, David; Dufva, Martin; Jensen, Thomas Glasdam; Kutter, Jörg Peter; Snakenborg, Detlef

Published in:

Journal of Micromechanics and Microengineering

Link to article, DOI:

10.1088/0960-1317/20/3/037001

Publication date:

2010

Document Version

Publisher's PDF, also known as Version of record

Link back to DTU Orbit

Citation (APA):

Sabourin, D., Dufva, M., Jensen, T. G., Kutter, J. P., \& Snakenborg, D. (2010). One-step fabrication of microfluidic chips with in-plane, adhesive-free interconnections. Journal of Micromechanics and Microengineering, 20(3), 037001. https://doi.org/10.1088/0960-1317/20/3/037001

\section{General rights}

Copyright and moral rights for the publications made accessible in the public portal are retained by the authors and/or other copyright owners and it is a condition of accessing publications that users recognise and abide by the legal requirements associated with these rights.

- Users may download and print one copy of any publication from the public portal for the purpose of private study or research.

- You may not further distribute the material or use it for any profit-making activity or commercial gain

- You may freely distribute the URL identifying the publication in the public portal 
One-step fabrication of microfluidic chips with in-plane, adhesive-free interconnections

This article has been downloaded from IOPscience. Please scroll down to see the full text article.

2010 J. Micromech. Microeng. 20037001

(http://iopscience.iop.org/0960-1317/20/3/037001)

View the table of contents for this issue, or go to the journal homepage for more

Download details:

IP Address: 192.38.67.112

The article was downloaded on 20/10/2011 at 15:24

Please note that terms and conditions apply. 


\title{
One-step fabrication of microfluidic chips with in-plane, adhesive-free interconnections
}

\author{
D Sabourin, M Dufva, T Jensen, J Kutter and D Snakenborg \\ DTU Nanotech, Department of Micro- and Nanotechnology, Technical University of Denmark (DTU), \\ Ørsteds Plads, Bygning 345Ø, DK-2800, Kongens Lyngby, Denmark \\ E-mail: detlef.snakenborg@nanotech.dtu.dk
}

Received 27 August 2009, in final form 1 December 2009

Published 15 February 2010

Online at stacks.iop.org/JMM/20/037001

\begin{abstract}
A simple method for creating interconnections to a common microfluidic device material, poly(methyl methacrylate) (PMMA), is presented. A press-fit interconnection is created between oversized, deformable tubing and complementary, undersized semi-circular ports fabricated into PMMA bonding surfaces by direct micromilling. Upon UV-assisted bonding the tubing is trapped in the ports of the PMMA chip and forms an integrated, in-plane and adhesive-free interconnection. The interconnections support the average pressure of $6.1 \mathrm{bar}$ and can be made with small dead volumes. A comparison is made to a similar interconnection approach which uses tubing to act as a gasket between a needle and port on the microfluidic chip.
\end{abstract}

S Online supplementary data available from stacks.iop.org/JMM/20/037001/mmedia

(Some figures in this article are in colour only in the electronic version)

\section{Introduction}

Increased adoption of microfluidic approaches can be encouraged by providing simple, low-cost, reliable and accessible fabrication methods and techniques. When beginning microfluidic work researchers often encounter difficulties establishing fluidic connections to devices. In this report a simple method for creating integrated interconnections to micromilled poly(methyl methacrylate) (PMMA) devices with deformable tubing is presented. PMMA is commonly used in microfluidic applications as it is highly amenable to fabrication by cost-effective and versatile methods such as micromilling, laser ablation, injection moulding and hot embossing. PMMA is of low cost, optically transparent and additionally it can readily be chemically modified or have biomolecules linked to its surface [1]. This report describes the creation of interconnections to PMMA microfluidic devices. Interconnections solutions for glass and silicon- [2-10] and PDMS- [11-18] based microdevices are described elsewhere and Fredrickson and Fan [19] provide a comprehensive review of interconnections.

Use of adhesives, such as epoxy, to create permanent and integrated interconnections by directly attaching needles and tubings to ports of microfluidic devices is usually regarded as the easiest method to create interconnections but suffers from drawbacks including risk of clogged channels, larger dead volumes resulting from manual alignment over small features. For multiple connections, this method can be time consuming and laborious. Methods to make permanent interconnections which prevent clogging have been proposed; however, not all are applicable to polymeric processing [8-10]. Solutions comparable to the method presented for creating interconnections to polymeric devices without adhesive use have been proposed.

Yang and Maeda [20, 21] used protruding silicone tubing to connect to the top surface of microfluidic devices placed in a socket. The protruding lengths of tubing act as $\mathrm{O}$ rings when pressed against the face of a microlfluidic chip. 


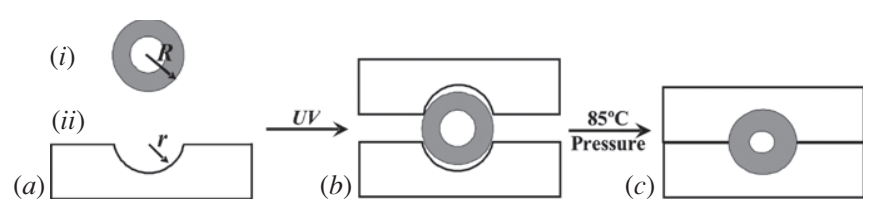

Figure 1. Schematic representation of the compressed tubing interconnection method. (a) (i) Cross-section of deformable tubing with outer radius $R$ and (ii) cross-section of one of two PMMA layers milled with ball mill to yield nearly semi-circular half-ports with radius $r$. Note that $R>r$. (b) PMMA surfaces were UV exposed and aligned above and below the tubing. Parts were then placed in a bonding press under pressure and at $85^{\circ} \mathrm{C}$ for $1 \mathrm{~h}$. (c) Cross-section of completed interconnection. Due to compression, the ID of the tubing has decreased.

Snakenborg et al [22] demonstrate in-plane interconnections using protruding soft tubing and also provide a theoretical treatment of this approach. Bhagat et al [23] fabricated a port clamp that used both O-rings and Teflon tubings to connect to the top surface of microfluidic devices made from cyclic olefin copolymer (COC). The Teflon tubing could be bent to create effectively in-plane interconnections. Perroziello et al [24] used thermal bonding to integrate O-rings into PMMA microfluidic chips. The microfluidic chip was then interfaced to a housing which contained vertically oriented metal ferrules with outer diameters (OD) greater than the inner diameter (ID) of the integrated O-ring. The mating of the chip with the housing resulted in a press-fit interconnection. Multiple, in-plane interconnections to polymeric devices using PDMS interconnection blocks cast from micromilled moulds were proposed by Sabourin et al [25]. The interconnection blocks contained multiple integrated channels bookended by O-ring like features. When a polymeric chip was placed between two interconnection blocks, the O-ring features are compressed and create fluidic connections to the polymeric chip. Though the above described approaches provide interconnection solutions, their implementation is more involved as they necessitate creation of additional components and assemblies apart from the microfluidic chip; they address including holders, moulds and clamps.

This technical note presents the research community with two methods for creating interconnections to PMMA. The first interconnection method presented creates a press-fit between readily available tubing and a PMMA microfluidic device during UV-assisted bonding. This is referred to as the compressed tubing interconnection. Leak pressures for this interconnection method are compared to a second press-fit method we commonly use referred to as the needle-tubing press-fit. This second method inserts an oversize needle into tubing with undersized ID. The tubing is housed in a port with the same OD as the tubing and as such the tubing acts as a gasket between needle and chip.

\section{Materials and methods}

\subsection{Interconnection design and fabrication}

Schematic representations of the compressed tubing and needle-tubing press-fit interconnection methods are shown in figures 1 and 3 respectively.

For the compressed tubing interconnection method two bonding surfaces of a PMMA chip were micromilled with ball end mills to yield 'half-ports' with semi-circular profiles (figure 1(a)). Ball end mills with diameters slightly smaller than the tubing OD were used. For this report, $2 \mathrm{~mm}$ ball end mills were used for $2.1 / 0.7 \mathrm{~mm}$ OD/ID silicone tubing (Reichelt Chemietechnik GmbH, Germany). Additionally, when milling the half-port features in the PMMA, the feature depth is set at less than half the OD (i.e. radius) of the tubing. Half-port features in each half of the PMMA test chips were milled to depths of either 0.90 or $0.95 \mathrm{~mm}$ such that when two surfaces are aligned and placed together for bonding, nearly circular ports of $1.8 \mathrm{~mm}$ or $1.9 \mathrm{~mm}$ diameter result. Both port sizes are thus undersized compared to the OD of the tubing. To bond PMMA chips, bonding surfaces were placed underneath a UV light source (DYMAX EC 5000 with p/n 36970 bulb, Torrington, Connecticut) for $60 \mathrm{~s}$ at a distance of about $12 \mathrm{~cm}$. As per the manufacturer's instructions, the light source was warmed up for $5 \mathrm{~min}$ prior to the exposure. After UV treatment (figure $1(b)$ ), the tubings were aligned to the halfports on one of the PMMA layers. The second PMMA layer was aligned bonding side down on top of the first layer and tubing (figure $1(b)$ ). The entire bonding assembly was placed in a bonding press $(\mathrm{P} / \mathrm{O} /$ Weber, Remshalden, Germany) with press heating plates set at $85{ }^{\circ} \mathrm{C}$ and an initial applied pressure of $1.0 \times 10^{5} \mathrm{kN} \mathrm{m}^{-2}$. After $1 \mathrm{~h}$ the heating plates were turned off and the bonding assembly was allowed to cool to room temperature in the press.

A total of 6 PMMA chips were made for testing. Three of the six chips were made by compressing $2.1 \mathrm{~mm}$ tubing into $1.8 \mathrm{~mm}$ ports and the other three were made by compressing $2.1 \mathrm{~mm}$ tubing into $1.9 \mathrm{~mm}$ ports. Each chip had eight separate interconnections (figure 2(a)). For both 1.8 and $1.9 \mathrm{~mm}$ chips, four of the eight interconnections had $3 \mathrm{~mm}$

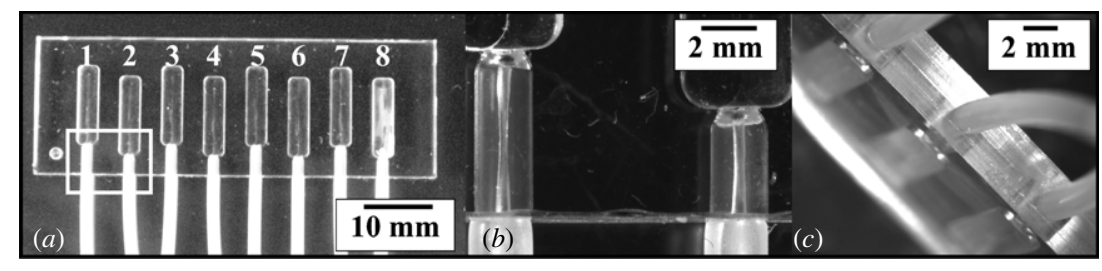

Figure 2. Compressed tubing interconnections. (a) PMMA chip used for pressure testing. Eight individual interconnections, assigned positions 1-8, were found on each chip. Tubing inserts of $5 \mathrm{~mm}$ were found in positions $1,3,5$ and 7 , and $3 \mathrm{~mm}$ tubing inserts were found in positions 2, 4, 6 and 8. A close-up of the area within the white box is shown in $(b)$. $(b)$ Top view of interconnection with 3 mm (left) and $5 \mathrm{~mm}$ (right) tubing inserts. $(c)$ Side view of PMMA chip showing in-plane interconnections. 
Table 1. Compressed tubing interconnection pressure data.

\begin{tabular}{ccclll}
\hline & & \multicolumn{3}{c}{ Pressure data } \\
\cline { 3 - 6 } $\begin{array}{l}\text { Port size } \\
\text { diameter (mm) }\end{array}$ & $\begin{array}{l}\text { Tubing insertion } \\
\text { length (mm) }\end{array}$ & $\begin{array}{l}\text { Average } \\
\text { (bar) }\end{array}$ & $\begin{array}{l}\text { SD } \\
\text { (bar) }\end{array}$ & $n$ & $\begin{array}{l}\text { Individual } \\
\text { values (bar) }\end{array}$ \\
\hline 1.8 & 3 & 4.4 & 2.0 & 12 & $6.1,6.1,3.1,2.1,0.9,0.9,0.6,0.6,6.1,6.1,6.1,0.6$ \\
1.8 & 5 & 0.8 & 0.1 & $11^{\text {a }}$ & $5.1,6.1,6.1,1.4,1.1,0.6,0.8,2.6,1.4,0.8,0.6,6.1$ \\
1.9 & 3 & 5.4 & 1.7 & 12 & $6.1,6.1,6.1,0.6,6.1,6.1,6.1,3.6,6.1,6.1,6.1,5.6$ \\
1.9 & 5 & 6.1 & 0 & $11^{\text {a }}$ & $6.1,6.1,6.1,6.1,6.1,6.1,6.1,6.1,6.1,6.1,6.1$ \\
\hline
\end{tabular}

a Two data points were excluded. For the first a premature leak occurred in the pressure measuring setup. For the second improper handling of chip following bonding cracked the PMMA above one chamber.

(a)
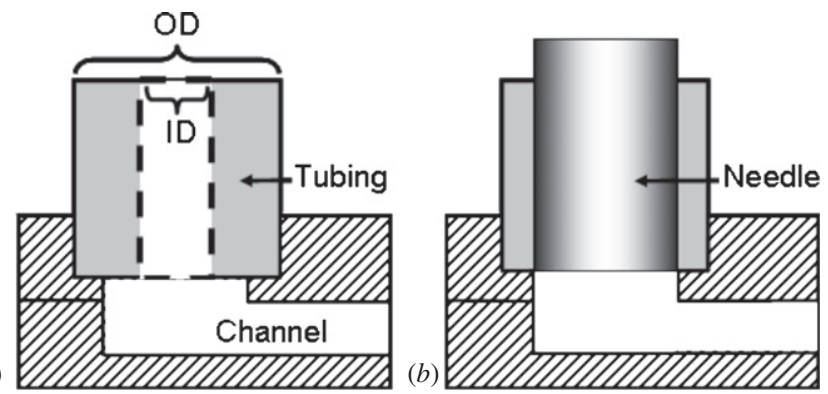

Figure 3. Needle-tubing press-fit interconnection. (a) Tubing with the same outer diameter (OD) as the circular port is inserted into the port directly above a microfluidic channel. $(b)$ A needle with a larger OD than the tubing's ID is inserted into the tubing. The tubing is compressed against both the needle and sidewall of the device. If the microfluidic channels dimensions and needle diameters are properly selected and aligned, small dead volumes can result [9].

long tubing inserts and the remaining four interconnections had $5 \mathrm{~mm}$ long tubing inserts (figures $2(a)$ and $(b)$ ). The 3 and $5 \mathrm{~mm}$ tubing inserts were altered (figure 2(a)). To facilitate and allow systematic investigation of failure modes each interconnection was assigned a position: $5 \mathrm{~mm}$ tubing inserts were in positions $1,3,5$ and 7 , and $3 \mathrm{~mm}$ tubing inserts were in positions 2, 4, 6 and 8 (figure $2(a)$ ).

For the needle-tubing press-fit approach (figure 3), tubing is placed in a port fabricated into the top surface of the microfluidic device. This port has the same OD as the tubing (figure 3(a)). A blunted syringe needle with larger OD than the tubing's ID is then inserted (figure $3(b)$ ) into the tubing. Compression of the tubing against both the sidewall and the needle creates a seal. To demonstrate this method, the same $2.1 \mathrm{~mm}$ OD silicone tubing (Reichelt Chemietechnik GmbH, Germany) with $0.7 \mathrm{~mm}$ ID was cut to $7 \mathrm{~mm}$ lengths with an angular cut of $1 \mathrm{~mm}$ pitch at one end. The angled cut facilitates placement of the tubing in the port. The angled end was inserted manually into $2.1 \mathrm{~mm}$ diameter port holes with $3.75 \mathrm{~mm}$ depth. The port holes were made by drilling a $5 \mathrm{~mm}$ thick PMMA plate using a micromilling machine (Folken, Glendale, California). Syringe needles with OD of $0.7,0.8$ and $0.9 \mathrm{~mm}$ were blunted and inserted into tubing pre-mounted in the PMMA plate holes.

\subsection{Leak pressure testing}

Leak pressures were determined visually and using a pressure sensor (Honeywell, Germany). For the compressed tubing interconnections (figure 2), observation through a microscope was used. For the needle-tubing press-fit method, microscopic observation was not required as leaks were sudden and violent, whereas for the compressed tubing method they were not. For compressed tubing interconnections, leaks were defined as the point at which a dye solution breached the outside of the tubing at the edge of the PMMA chip (figure 2(c)). The dye solution was pumped from a syringe pump to the interconnection being tested. Steps were taken to limit the amount of air in the system as interconnections were tested in chips with closed channels. For the needle-tubing press-fit method (figure 3), a new piece of tubing and a new needle were used for each measurement. The needle-tubing press-fit method test values were collected first. As a result of observed leaks in the pressure measuring setup during testing of the needle-tubing press-fit method above 6.1 bar, tests for the compressed tubing method were voluntarily stopped at 6.1 bar. This value is three times greater than the 2 bar pressure limit described as applicable to most microfluidic applications [23]. For the needle-tubing press fit method, in cases where the test setup leaked the final pressure recorded was taken as the test value.

\section{Results and discussion}

\subsection{Compressed tubing interconnections}

The compressed tubing method simultaneously yields the finishing of the PMMA chip and the integration of in-plane interconnections during the bonding process. UV radiation reduces the glass transition temperature of polymer's surface layer, only several microns deep, without changing the glass transition temperature of the bulk polymer [26, 27]. Structures can then be bonded above the glass transition temperature of the surface layer but below that of the bulk PMMA layer. Sealing is thus permitted without a loss in structure quality.

Visual inspection of the compressed tubing interconnection test chips indicated that the bond quality of chips with $1.9 \mathrm{~mm}$ ports was superior to that when using $1.8 \mathrm{~mm}$ ports (figure 4). Areas where the two PMMA layers were not well bonded were seen adjacent to the interconnections on $1.8 \mathrm{~mm}$ port chips (figure $4(b)$ ). For $1.8 \mathrm{~mm}$ port a physical limitation was reached where tubing could not consistently be compressed within the port opening during the bonding process. Bond quality suffered and, as a result, so did interconnection performance (table 1). The compression of the tubings affected the geometry of the channels inside the tubings. Tubing channels were more constricted for $1.8 \mathrm{~mm}$ port chips versus $1.9 \mathrm{~mm}$ port chips, 


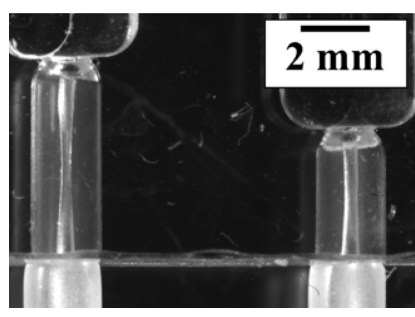

(a)

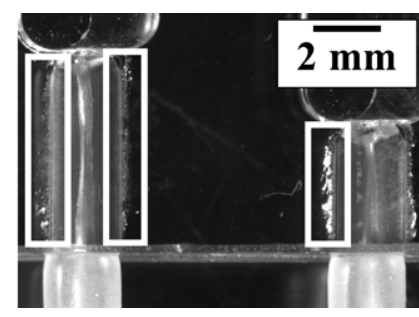

(b)
Figure 4. Bond quality of compressed tubing interconnections. (a) $1.9 \mathrm{~mm}$ port interconnections for a $5 \mathrm{~mm}$ (left) and $3 \mathrm{~mm}$ (right) long tubing insert. $5 \mathrm{~mm}$ tubing inserts were more constricted than $3 \mathrm{~mm}$ tubing inserts. (b) $1.8 \mathrm{~mm}$ port interconnections for a $5 \mathrm{~mm}$ (left) and $3 \mathrm{~mm}$ (right) long tubing insert. Boxes indicate areas representative of poor bonding.

and for 5 versus $3 \mathrm{~mm}$ inserted tubing lengths (figure 4); however, no blocked channels were observed.

Table 1 presents results from a first test cycle of individual interconnections on chips with 1.8 or $1.9 \mathrm{~mm}$ ports and 3 or $5 \mathrm{~mm}$ tubing inserts. $1.9 \mathrm{~mm}$ port interconnections yielded higher and more consistent pressure performance as a result of better bond quality (table 1 ). For $1.8 \mathrm{~mm}$ ports, data scatter correlated to bonding quality (figure $4(b)$ ).

For $1.9 \mathrm{~mm}$ ports, three values were recorded below the maximum 6.1 bar: 0.6, 3.6 and 5.6 bar. All three values came from position 8 , a $3 \mathrm{~mm}$ long tubing insert, on the three separate $1.9 \mathrm{~mm}$ port sized chips. Inspection of position 8 for $1.9 \mathrm{~mm}$ port chips showed a clearly reduced bond quality. Position 8 also yielded the lowest pressure values for the three $1.8 \mathrm{~mm}$ port chips. A systematic failure in bonding, e.g. caused by an uneven distribution of bonding forces, is attributed as a cause and suggests that visual inspection of the bond quality prior to the usage is strongly advisable. Given the systematic failure at position 8 , if these data points are removed, there is no difference in pressure limits for $1.9 \mathrm{~mm}$ ports with 3 or $5 \mathrm{~mm}$ long tubing insertions as both are tested with an average of 6.1 bar and SD of 0. However, a shorter tubing insert is probably preferable as the tubings were less constricted and less chip space was used (figure 4).

Test devices with multiple, as opposed to a single, interconnection were used to validate the pressure limits of the compressed tubing method. This is representative of microfluidic chips which need multiple reagents to be added to one reactor, or employ hydrodynamic focusing or in cases where a single chip contains many parallel but individually addressable fluidic networks. Importantly, as the failure of a single interconnection below a required design pressure threshold usually results in yield loss, system downtime and ultimately a lost experiment, it is important that the lowest expected pressure values are well above the threshold. Insertion of the $2.1 \mathrm{~mm}$ tubing $1.9 \mathrm{~mm}$ ports (table 1) consistently provided performance three times greater than the threshold of 2 bar applicable for most microfluidic applications. Owing to the pressure test setup limitations, it is likely that leak pressures for the $1.9 \mathrm{~mm}$ port size conditions are underestimated.

Re-usability and robustness to handling from repeated connection and removal from the test system was demonstrated
Table 2. Needle-tubing press-fit pressure data.

\begin{tabular}{ccclcl}
\hline & & \multicolumn{4}{c}{ Pressure data } \\
\cline { 3 - 6 } $\begin{array}{l}\text { Tubing } \\
\text { OD (mm) }\end{array}$ & $\begin{array}{l}\text { Needle } \\
\text { OD (mm) }\end{array}$ & $\begin{array}{l}\text { Average } \\
\text { (bar) }\end{array}$ & $\begin{array}{l}\text { SD } \\
(\text { bar })\end{array}$ & $\begin{array}{l}\text { Individual } \\
\text { values (bar) }\end{array}$ \\
\hline \multirow{2}{*}{2.1} & 0.7 & 0 & 0 & 2 & 0,0 \\
& 0.8 & 6.4 & 0.6 & 5 & $6.1,5.7,7.4,6.1,6.4$ \\
& 0.9 & 7.4 & 0.6 & 4 & $6.3,7.1,7.8,6.9$ \\
\hline
\end{tabular}

by repetitive testing of a $1.9 \mathrm{~mm}$ port feature chip. Individual interconnections were tested up to four more additional times and all pressure readings reached 6.1 bar. Further insertion cycles could have been tested but due to PMMA's properties, including low cost, we consider this material most suitable for one-time use and/or rapid prototyping.

\subsection{Needle-tubing press-fit}

Tubing pieces and blunted needles were easily introduced into pre-fabricated holes and the tubing, respectively. Results for this method are shown in table 2 . The reader may recall that these values were collected prior to values for the compressed tubing method and that following the observation of leaks in the measuring setup the voluntary stop limit of 6.1 bar was used for the compressed tubing method. Therefore, in some cases values reported in table 2 are above this point.

Leaks occurred immediately for $0.7 \mathrm{~mm}$ OD needle inserts. For $0.8 \mathrm{~mm}$ needle inserts, the average leak pressure was 6.4 bar with a maximum and minimum of 7.4 and 5.7 bar. For $0.9 \mathrm{~mm}$ needle inserts the average leak pressure was 7.0 bar with a maximum and minimum of 7.8 and 6.3 bar. Values for $0.9 \mathrm{~mm}$ needles are likely underestimated due to aforementioned leaks in the test setup. The needle-tubing press-fit method creates connections quickly and supports pressures well above the 2 bar pressure limit applicable to most microfluidic applications [23] and should work equally well with other polymers. Additionally, it performs equally well as a similar method reporting maximum pressure of 5.6 bar but which uses epoxy to affix syringe needles into polymeric ports [28].

\subsection{Comparison of methods}

Both interconnection methods are easily implemented, use readily available materials, are adhesive-free and yield seal performances well above 2 bar (tables 1 and 2) threshold. The tubing size used here was selected for ease of handling. In our experience, handling tubings less than $2 \mathrm{~mm}$ in diameter can be cumbersome. Both interconnection methods are applicable to other tubing sizes and initial experiments should quickly provide indication of suitable pairing of over- and undersized elements which provide suitable pressure performance and device yield.

The needle-tubing method ensures very consistent flow resistances from one interconnection to the next. As with other interconnection methods, especially those using glues, epoxies and/or adhesives, the compressed tubing method can introduce flow resistance variations within the interconnection 
Table 3. Interconnection methods comparable to compressed tubing.

\begin{tabular}{|c|c|c|c|c|c|}
\hline Method & General description & $\begin{array}{l}\text { Pressure } \\
\text { limits (bar) }{ }^{\mathrm{a}}\end{array}$ & $\begin{array}{l}\text { Number of } \\
\text { interconnections }\end{array}$ & In-plane & Ref. \\
\hline \multirow[t]{3}{*}{ Protruding soft tubing } & Silicone tubing compressed to top face & 2 & 10 & $y^{\mathrm{b}}$ & {$[21]$} \\
\hline & Silicone tubing compressed to top face & 2 & 20 & $y^{\mathrm{b}}$ & [20] \\
\hline & Silicone tube compressed to side face & 12.3 & 1 & $y$ & [22] \\
\hline \multirow{3}{*}{ O-ring } & O-rings heat annealed within PMMA chip & 7.5 & 4 & $n$ & [24] \\
\hline & O-rings compressed to top face/press-fit with tubing & 17 & 3 & $y^{\mathrm{b}}$ & {$[23]$} \\
\hline & $\begin{array}{l}\text { Chip placed between PDMS connecting blocks with } \\
\text { integrated channels and O-ring features }\end{array}$ & 5.5 & 24 & $y$ & {$[25]$} \\
\hline
\end{tabular}

${ }^{a}$ Due to differences in test methods and reporting of data, values reported are either average or maximum values. The reader should consult the appropriate reference for details concerning testing.

$\mathrm{b}$ Though the connection is made to the top face, tubings used with these methods could be bent to effectively become in-plane.

resulting from differences in the tubing constriction (figure 4). Whether or not this is problematic is both dependent on the application and the method used to drive the fluidic device. Devices driven by pressure sources can be affected by the difference in constriction; however, constant volume displacement pumps negate these differences as fluid velocities and volumetric displacements will be the same within equally sized microfluidic channels located after the interconnections.

Both interconnection methods can result in small dead volumes. The fabrication of high quality microfluidic chips from multiple layers requires proper alignment of layers which in turn reduces dead volumes. Two simple alignment strategies can be implemented: (i) machining aligned holes within the microfluidic chip layers capable of housing alignment pins, or (ii) to machining materials such as aluminium to create bonding assemblies. As used in this study, and in our general experience, it is easy to fabricate aluminium sheets containing holes which house alignment pins coincident with the periphery of the microfluidic chip to provide alignment. With proper chip layer alignment and features as shown in figure 3, and according to the analysis by Puntambekar et al [9], the needle-tubing press fit method can yield effectively zero dead volumes.

To minimize dead volumes in the compressed tubing method, tubing must be aligned next to the microfluidic channel entrance. The semi-spherical geometries shown in figures 2 and 4 do not allow such placement; however, these are not representative of channel architectures we would use for applications requiring small dead volumes. The semispherical geometries are present because the pressure test microfluidic chips were fabricated using minimum milling steps and tool changes. The semi-spherical geometries can be eliminated with further milling steps which can create geometries highly complementary to the tubing shape. Doing so will allow tubing to be brought next to the microfluidic channel and reduce dead volumes. For dimensions and materials used in this study, the estimated dead volume for tubing aligned to the edge of the semispherical geometry and where a $100 \times 100 \mu \mathrm{m}$ is used as the microfluidic channel following the interconnection channel is $1.9 \mu \mathrm{L}$ (see supplementary information, available at stacks.iop.org/JMM/20/037001/mmedia). We consider this an estimate of maximum dead volume as (i) figures 2 and 4 show that tubing is compressed into this semi-spherical area, thereby reducing the dead volume, and (ii) a $100 \times 100 \mu \mathrm{m}$ channel is representative of the small end of channel dimensions created by micromilling. The compressed tubing procedure (section 2.1) provides feedback to the user with respect to placing tubing next to microfluidic channels. Following UV exposure, the bottom PMMA layer was placed on the bottom half of the aluminium bonding assembly which had alignment pins extending upwards. Tubing was then aligned to the halfports on the bottom PMMA layer and the alignment pins ensured when the top PMMA layer was brought down on top of bottom layer, the features were aligned. When only lightly pressing the top PMMA layer to the bottom PMMA layer, and before placing the top aluminium layer on the assembly and into the bonding press, tubing could be slid towards the end of the interconnection channel. The user could then determine when tubing could not be further pushed towards the microfluidic channel. The transparent nature of PMMA also allows visual inspection. Therefore, for materials and dimensions used in this study, noting that (i) further milling steps can remove the semi-spherical geometries, (ii) a simple bonding assembly provides tubing alignment feedback to the user, (iii) as shown in figures 2 and 4, tubing is pressed towards the microfluidic channel during the bonding process, and (iv) if the IDs of the tubing and corresponding microfluidic channel are matched, we estimate that dead volumes of approximately $300 \mathrm{~nL}$ (see supplementary information, available at stacks.iop.org/JMM/20/037001/mmedia).

The compressed tubing method offers several advantages. Interconnections are formed while the chip is bonded allowing immediate testing and thus avoiding unnecessary downstream processing and/or machining steps. When assembling the PMMA devices following the UV exposure, the tubings provide self-alignment between layers. While the needletubing press fit requires additional post-fabrication handling of the microfluidic device, i.e. needle insertion, which could damage and place stress on the device, the compressed tubing method avoids this. When connecting devices using the compressed tubing approach, needles interface only to the tubing end not within the device (figure 2). The compressed tubing approach forms in-plane interconnections better suited to microscopic observation (figure 2) than the needle-tubing press-fit interconnections, which are usually oriented vertically (figure 3 ) to simplify device fabrication. 
Methods comparable to the compressed tubing approach are presented in table 3 . The pressure performance of the $1.9 \mathrm{~mm}$ ports is within the range of the methods found in table 3. Yang et al $[20,21]$ used $1 \mathrm{~mm}$ OD tubings to create interconnections to the top surface of microfluidic chip with interconnection pitches of $2.2 \mathrm{~mm} \mathrm{[21]} \mathrm{or} 2.5 \mathrm{~mm} \mathrm{[20]} \mathrm{and}$ Sabourin et al [25] report pitch of $2.25 \mathrm{~mm}$ using cast PDMS interconnection blocks. Had similar tubing sizes been used in this report, similar interconnection pitches to these methods are possible to achieve. The use of a holder or bonding assembly permitting placement and alignment of tubing is helpful when creating many interconnections or interconnections with similar pitch as those reported. Additionally, the compressed tubing method does not require side surfaces of microfluidic chips to be polished following bonding $[22,25]$ in order to obtain leak-free interconnections and accommodates slight misalignments between bonding layers. Methods that use holders or clamps [22-25] to create interconnections also dictate chip size and interconnection location. The compressed tubing method is flexible with respect to chip size and interconnection location. Though solutions using holders and clamps facilitate rapid chip testing, the compressed tubing method negates this advantage as interconnections are formed with the chip and tubing, to be hooked up to pumps, etc, is already present.

\subsection{Example applications for the compressed tubing method}

The compressed tubing method presented is a general method for creating in-plane, adhesive-free interconnections. Though presented for PMMA devices, for polymeric materials for which UV-assisted bonding is not possible, thermal or chemical bonding processes [27] could be used to implement the general approach.

A method which does not incorporate adhesive use to form interconnections is of great advantage as it avoids potential issues related to sensitivity and biocompatibility [29]. We will be using the $1.9 \mathrm{~mm}$ port compressed tubing interconnections for investigations related to parallel analysis of microarray hybridization and cell cultures within PMMA devices. The architecture of these devices will closely resemble the PMMA chips presented in figure 2 , but with each chamber having both an inlet and an outlet. These applications require pressures much lower than the 6 bar reported. The compressed tubing is well suited to these applications. In particular the in-plane connections afford unobstructed microscopic observation.

\section{Conclusions}

Both interconnection methods presented are simple to implement. The compressed tubing methods eliminate additional processing steps while rapid, immediate testing is enabled resulting in in-plane interconnections better suited to microscopic observation. The achieved results indicate a strong correlation between bond quality and performance. The use of $0.7 \mathrm{~mm} / 2.1 \mathrm{~mm}$ ID/OD tubing with a $1.9 \mathrm{~mm}$ port feature created by micromilling PMMA with a $2 \mathrm{~mm}$ ball mill yields interconnections which consistently show leakfree performance up to 6.1 bar, well above the 2 bar threshold considered applicable for most microfluidic applications.

\section{Acknowledgments}

The authors acknowledge funding from a DTU Ph D student grant, the Danish Strategic Research Council (NABIIT programme ProCell) and the FP7-EXCELL.

\section{References}

[1] Chen Y, Zhang L and Chen G 2008 Fabrication, modification and application of poly(methyl methacrylate) microfluidic chips Electrophoresis 29 1801-14

[2] Gonzalez C, Collins SD and Smith RL 1998 Fluidic interconnects for modular assembly of chemical microsystems Sensors Actuators B 49 40-5

[3] Gray BL, Collins SD and Smith RL 2004 Interlocking mechanical and fluidic interconnections for microfluidic circuit boards Sensors Actuators A 112 18-24

[4] Gray BL et al 1999 Novel interconnection technologies for integrated microfluidic systems Sensors Actuators A 77 57-65

[5] Lee ES, Howard D, Liang E, Collins SD and Smith RL 2004 Removable tubing interconnects for glass-based micro-fluidic systems made using ECDM J. Micromech. Microeng. 14 535-41

[6] Meng E, Wu SY and Tai YC 2001 Silicon couplers for microfluidic applications Fresenius' J. Anal. Chem. $371270-5$

[7] Pan T, Baldi A and Ziaie B 2006 A reworkable adhesive-free interconnection technology for microfluidic systems J. Microelectromech. Syst. 15 267-72

[8] Pattekar AV and Kothare MV 2003 Novel microfluidic interconnectors for high temperature and pressure applications J. Micromech. Microeng. 13 337-45

[9] Puntambekar A and Ahn CH 2002 Self-aligning microfluidic interconnects for glass- and plastic-based microfluidic systems J. Micromech. Microeng. 12 35-40

[10] Tsai JH and Lin LW 2001 Micro-to-macro fluidic interconnectors with an integrated polymer sealant J. Micromech. Microeng. 11 577-81

[11] Chiou CH and Lee GB 2004 Minimal dead-volume connectors for microfluidics using PDMS casting techniques J. Micromech. Microeng 14 1484-90

[12] Christensen AM, Chang-Yen DA and Gale BK 2005 Characterization of interconnects used in PDMS microfluidic systems J. Micromech. Microeng. 15 928-34

[13] Dalton C and Kaler K 2007 A cost effective, re-configurable electrokinetic microfluidic chip platform Sensors Actuators B 123 628-35

[14] Jo B H, Van Lerberghe L M, Motsegood K M and Beebe D J 2000 Three-dimensional micro-channel fabrication in polydimethylsiloxane (PDMS) elastomer J. Microelectromech. Syst. 9 76-81

[15] Li SF and Chen SC 2003 Polydimethylsioxane fluidic interconnects for microfluidic systems IEEE Trans. Adv. Packag. 26 242-7

[16] McDonald J C and Whitesides G M 2002 Poly(dimethylsiloxane) as a material for fabricating microfluidic devices Acc. Chem. Res. 35 491-9

[17] Quaglio M et al 2008 Evaluation of different PDMS interconnection solutions for silicon, Pyrex and COC microfluidic chips J. Micromech. Microeng. 18055012

[18] Saarela V et al 2006 Re-usable multi-inlet PDMS fluidic connector Sensors Actuators B 114 552-7 
[19] Fredrickson CK and Fan ZH 2004 Macro-to-micro interfaces for microfluidic devices Lab Chip 4 526-33

[20] Yang Z and Maeda R 2002 A world-to-chip socket for microfluidic prototype development Electrophoresis 23 3474-8

[21] Yang Z and Maeda R 2003 Socket with built-in valves for the interconnection of microfluidic chips to macro constituents J. Chromatogr. A 29-33

[22] Snakenborg D, Perozziello G, Geschke O and Kutter JP 2007 A fast and reliable way to establish fluidic connections to planar microchips J. Micromech. Microeng. 17 98-103

[23] Bhagat A A S, Jothimuthu P, Pais A and Papautsky I 2007 Re-usable quick-release interconnect for characterization of microfluidic systems J. Micromech. Microeng. 17 42-9

[24] Perozziello G, Bundgaard F and Geschke O 2008 Fluidic interconnections for microfluidic systems: a new integrated fluidic interconnection allowing plug 'n' play functionality Sensors Actuators B 130 947-53
[25] Sabourin D, Snakenborg D and Dufva M 2009 Interconnection blocks: a method for providing reusable, rapid, multiple, aligned and planar microfluidic interconnections J. Micromech. Microeng. 19035021

[26] Truckenmüller R, HENZI P, Herrmann D, Saile V and Schomburg W K 2004 Bonding of polymer microstructures by UV irradiation and subsequent welding at low temperatures Microsyst. Technol. $10372-4$

[27] Tsao C W and Devoe D L 2009 Bonding of thermoplastic polymer microfluidics Microfluidics Nanofluidics 6 1-16

[28] Korivii NSaJ L 2007 A generic chip-to-world fluidic interconnect system for microfluidic devices IEEE 39th Southeastern Symp. on System Theory (Macon, GA, 04-06 March) 176-80

[29] Stangegaard M, Petronis S, Jorgensen A M, Christensen C B and Dufva M 2006 A biocompatible micro cell culture chamber (microCCC) for the culturing and on-line monitoring of eukaryote cells Lab Chip 6 1045-51 